\title{
The relation of ongoing brain activity, evoked neural responses, and cognition
}

\author{
Sepideh Sadaghiani 1,2,3*, Guido Hesselmann ${ }^{4}$, Karl J. Friston ${ }^{5}$ and Andreas Kleinschmidt ${ }^{1,2}$ \\ 1 Institut National de la Santé et de la Recherche Médicale Unité 992 Cognitive Neuroimaging Unit, Gif-sur-Yvette, France \\ 2 NeuroSpin, I2BM, DSV, CEA, Gif-sur-Yvette, France \\ 3 International Max Planck Research School of Neural and Behavioural Sciences, University of Tübingen, Tübingen, Germany \\ ${ }^{4}$ Department of Neurobiology, Weizmann Institute of Science, Rehovot, Israel \\ ${ }^{5}$ Wellcome Trust Centre for Neuroimaging, University College London, London, UK
}

Edited by:

Lucina Q. Uddin,

Stanford University, USA

Reviewed by:

Mike Fox, Partners Healthcare, USA

Gordon Shulman, Washington

University School of Medicine, USA

*Correspondence:

Sepideh Sadaghiani, Institut National de la Santé et de la Recherche

Médicale Unité 992, NeuroSpin, CEA

SAC/DSV/I2BM, Bât 145, Point Courrier

156, F-91191 Gif-sur-Yvette, France.

e-mail: sepideh.sadaghiani@gmail.com
Ongoing brain activity has been observed since the earliest neurophysiological recordings and is found over a wide range of temporal and spatial scales. It is characterized by remarkably large spontaneous modulations. Here, we review evidence for the functional role of these ongoing activity fluctuations and argue that they constitute an essential property of the neural architecture underlying cognition. The role of spontaneous activity fluctuations is probably best understood when considering both their spatiotemporal structure and their functional impact on cognition. We first briefly argue against a "segregationist" view on ongoing activity, both in time and space, which would selectively associate certain frequency bands or levels of spatial organization with specific functional roles. Instead, we emphasize the functional importance of the full range, from differentiation to integration, of intrinsic activity within a hierarchical spatiotemporal structure. We then highlight the flexibility and context-sensitivity of intrinsic functional connectivity that suggest its involvement in functionally relevant information processing. This role in information processing is pursued by reviewing how ongoing brain activity interacts with afferent and efferent information exchange of the brain with its environment. We focus on the relationship between the variability of ongoing and evoked brain activity, and review recent reports that tie ongoing brain activity fluctuations to variability in human perception and behavior. Finally, these observations are discussed within the framework of the free-energy principle which - applied to human brain function - provides a theoretical account for a non-random, coordinated interaction of ongoing and evoked activity in perception and behavior.

Keywords: ongoing activity, spontaneous activity, resting state functional connectivity, intrinsic functional connectivity, fMRI, prestimulus activity, variability, fluctuations

\section{INTRODUCTION}

Our review is based on the premise that - just as man-made architectures (and probably even more so) - the nature of biological systems is best understood by jointly considering their form and function. We will attempt to apply this view to ongoing brain activity. Our review of the form of ongoing or "spontaneous" brain activity will cover its temporal and spatial structure. Instead of attempting to be exhaustive in this respect, we will selectively emphasize some aspects mainly for two reasons; first, because we feel they may be under-represented in a field that is currently dominated by the notion of "resting state networks"; second, because we feel that these aspects are helpful when pondering the function of ongoing activity. In the second part of our review, function will then be the theme developed in more detail. We will focus on cognitive consequences of ongoing activity fluctuations, for the simple reason that they permit the most direct probes of functional significance for a phenomenon that is no longer fully "spontaneous" when bound into a context so as to measure function. Across this analysis of form and function we will then discuss how one theoretical framework, that of "free energy" introduced by one of us (Friston, 2005), may provide important clues for understanding the nature of ongoing brain activity. Our review will mainly concentrate on the human brain and functional MRI. The latter has been informative due to its superb localizing power and its exquisite capability to record the dynamics of neuronal population activity across the entire brain and to hence capture large-scale functional connectivity patterns. Yet, for instance when addressing temporal properties as below, limitations of hemodynamic signals will lead us to also discuss electrophysiological findings as well as observations relying on invasive procedures that cannot usually be applied in human subjects.

\section{THE TEMPORAL STRUCTURE OF ONGOING BRAIN ACTIVITY}

One of the most prominent features of ongoing activity is the fact that it fluctuates over time. This in itself can give rise to interesting speculations regarding function. If one thinks of a car engine, where such behavior would be functionally deleterious, one might wonder whether, and if so how, such fluctuations can be associated with a functional benefit (McDonnell and Abbott, 2009). Let us first consider the formal properties of these temporal fluctuations.

Ongoing human brain activity recorded by local electrocorticography (Freeman et al., 2000) shows a power law scaling but also an embedding of discrete peaks reflecting band-limited oscillatory activity. Interestingly, power in these distinct frequency bands is in turn also modulated over time with a predominance of very slow 
frequencies (Leopold et al., 2003; Nir et al., 2008). Descriptively, it has been shown that there is a coupling or nesting of the higherfrequency electrical activity into the infra-slow (usually defined as $<0.1 \mathrm{~Hz}$ ) fluctuations (Vanhatalo et al., 2004; He et al., 2010) but the mechanisms and directionality of this relation are not yet fully understood. Studies comparing invasive electrophysiological recordings with functional neuroimaging results have obtained evidence of coupling between hemodynamic signals and both slow cortical potentials (He et al., 2008) as well as power of high-frequency bandlimited activity, both evoked and spontaneous (Nir et al., 2007; Shmuel and Leopold, 2008).

We conclude from these observations that the temporal properties of ongoing activity can serve to warn us against a preoccupation with the "millisecond range" when studying brain function. Yet, assuming a conservative stance, we also conclude that there is currently no reason for a rebound into a view where infra-slow fluctuations in a specific frequency range could be considered a distinct entity of neural processes, other than those active in the processing of, for instance, sensory events. Studies using fMRI have established an apparent predominance of slow fluctuations in ongoing brain activity but there are several caveats to be borne in mind. First, the issue of whether the actual neural activity reflected in the hemodynamic signals shows power law scaling as in electrical recordings is still being debated (Cole et al., 2010). It is certain that the low pass filter characteristics of hemodynamic signals only permit tracking of slow neural activity modulations, cutting off little above the range of the infra-slow frequencies. And there are additional concerns related to the fact that - in spite of quantitative differences - even "BOLD signal" variations from a water phantom can readily manifest power law scaling due to properties of the MRI scanner (Zarahn et al., 1997; but see also Fox et al., 2007). It has also not been established that the spatial pattern of functional connectivity depends on the temporal scale under consideration, other than obvious effects related to signal power.

Together, we suggest thinking of the presently available evidence as an indication that brain activity over time may display at least partially scale-invariant characteristics. Such pink noise or power law scaling is not a privilege of the brain or even of biological systems but a feature of many if not all complex systems (Mandelbrot, 1998). Its ubiquitous presence does not denigrate its importance though. Regarding the brain, several researchers have emphasized the importance of this temporal structure for endowing neural processes with an inherent long-term memory (Linkenkaer-Hansen et al., 2001; Buzsáki, 2006). The memory function in this view does not reside in a specific frequency range but merely has a holistic pattern. However, for an alternative opinion and a more differentiated discussion of these issues we refer readers to a recent review by Raichle (2010).

\section{THE SPATIAL STRUCTURE OF ONGOING BRAIN ACTIVITY}

Our main point in the previous section was to review the literature that safeguards us against a temporal "segregationist" view. We believe that there is a similar danger in the spatial domain. The reason why many laboratories have focused on infra-slow fluctuations is that due to their power and their at least partial distinctness from other, namely "noise" signal sources in functional neuroimaging, these fluctuations have proven useful for studying the spatial structure of ongoing brain activity. Such functional connectivity studies have also focused on the only paradigm which permits an apparently unambiguous assignment of signal variations to ongoing brain activity; namely, the "resting state". Despite concerns about contributions from technical and physiological noise, the rationale of these socalled resting-state functional connectivity studies has been validated by concurrent electrophysiological recordings. In particular, it has been established that slow fluctuations in power of band-limited oscillations can be directly linked to the ongoing activity fluctuations observed with fMRI (Shmuel and Leopold, 2008) and involve similar distributed spatial structures (Laufs et al., 2003).

With this functional imaging approach, it is now well established that spontaneous brain activity fluctuations are spatially organized into a largely reproducible structure. The emphasis in a (large) literature, whose review is beyond the scope of this article, has been to define anatomically such resting-state or intrinsic functional connectivity networks (ICNs). We will adopt the latter terminology because there is reason to believe that similar correlational structures persist even when subjects are exposed to vivid sensory stimulation (Golland et al., 2007). The definition of ICNs revolves essentially around two issues, that of constituent regions and that of boundaries. And this definition has relied on two approaches, one hypothesisdriven as exemplified in analysis of functional connectivity with a so-called seed region (e.g., Biswal et al., 1997; Greicius et al., 2003), the other data-driven as exemplified by independent component analyses (e.g., Beckmann et al., 2005). The ultimate goal of these analyses is to derive an anatomical segregation from the recordings of ongoing brain activity fluctuations. Notwithstanding a great degree of convergence and robustness across many different laboratories, both of these approaches have proven to be heavily influenced by user-dependent settings. What such settings usually express is the user's expectation regarding the degree of modularity in ongoing brain activity. While some laboratories emphasize big dichotomies (e.g., Fox et al., 2006b; Golland et al., 2008), others seek to establish a fine-grained differentiation (e.g., Margulies et al., 2007).

In this context, we would like to emphasize that the actual data structure does not suggest clear-cut modularity but only a gradual differentiation. The reason for such graded modularity is that the correlational structure of ongoing activity is bound together in a hierarchy. This structure is probably best thought of as a tree with the underlying activity correlations displaying a hierarchy from global to local levels (Ferrarini et al., 2009). These levels of organization range from the entirety of gray matter as the trunk, over systems of regions as the branches to within-region correlations as the foliage (Marrelec et al., 2008; Meunier et al., 2009). In fact, the strong presence of variance shared across all local levels and reflected in global gray matter (Schölvinck et al., 2010) correlation has led to considerable confusion regarding the degree of diversification or antagonism that can be observed across different ICNs (Fox et al., 2009; Murphy et al., 2009). ICNs can be considered a mid-level cross-section of this hierarchical tree where regions within an ICN share a lot of variance and where this variance is sufficiently distinct from that expressed in other ICNs to draw a separating line. As a function of whether one emphasizes similarity or distinctness of local variations in ongoing activity, data-driven analyses will produce quite different numbers of ICNs (e.g., Varoquaux et al., 2010). In our metaphor, this corresponds to the distance of the cross-section from the ground. 
As a consequence of the hierarchical organization of ongoing activity, raising the level of cross-section higher from the ground will yield more fine-grained subdivisions of networks both at anatomical and functional connectivity levels. As an example, the postero-medial part of the most extensively studied ICN, the default-mode network, has recently been subdivided into three precuneus parts and a posterior cingulate part on the basis of distinct large-scale intrinsic connectivity patterns, each of which suggest different functional roles (Margulies et al., 2009). Another example involves the difficulty in anatomical and functional definition of the so-called "task-positive" system. An initially useful step was to distinguish the "task-negative" default-mode ICN from "taskpositive" regions, the latter referring to a large set of regions showing activation in most types of cognitive paradigms (Fox et al., 2005). Using seed regions in the dorsal attention network, the resulting intrinsic connectivity system was not confined to the dorsal attention system as defined in paradigm-based studies (Corbetta and Shulman, 2002) but due to shared variance also included anterior insula/frontal operculum, anterior prefrontal cortex, and inferolateral parietal and frontal areas. These additional areas partially overlap with an added ICN, termed the fronto-parietal control system conceptualized to serve cognitive control (Vincent et al., 2008). Conversely, other studies dissected cognitive control functions into two distinct ICNs, a cingulo-insular-thalamic and a lateral parieto-frontal network for sustained vs. adaptive/executive cognitive control, respectively (Dosenbach et al., 2006, 2007; Seeley et al., 2007). These findings clarify that the hierarchically embedded levels of spatial structure in intrinsic connectivity range down to sub-network and ultimately sub-region correlations. In fact, albeit on a different temporal scale, such patterns can even be recovered within single areas, and align with their mesoscopic functional architecture (Kenet et al., 2003).

Over and above the issue of modularity, defining ICNs in terms of anatomical boundaries has also proven difficult. This difficulty is largely due to the fact that "networks" are not clear-cut and rigid sets of constituent regions. Rather, the term "network" should be thought of as a gradual clustering according to a similar activity profile. As such, this term can of course help to interpret, communicate and compare experimental results but should not mislead to consider networks as strictly segregated. The spatial patterns are susceptible to precise positioning of seed regions and it has for instance been demonstrated that there are fairly smooth transitional zones between ICNs (Cohen et al., 2008). Even though some of these difficulties may be due to the intrinsic spatial smoothness of hemodynamic signals rather than underlying neural architecture, such observations may account for observed discrepancies. With respect to the task-positive regions however, these difficulties also stem from the existence of an ensemble of several interconnected task-positive ICNs. Accordingly, the labeling issue becomes most critical for connection hubs such as the anterior insula (Sterzer and Kleinschmidt, 2010) which has been suggested to orchestrate activity across different ICNs (Sridharan et al., 2008). In addition to the ICNs discussed above, the anterior insula has also been characterized as a major node in a right-lateralized ventral attention system (Eckert et al., 2008). This latter system (Fox et al., 2006b) in turn widely overlaps with the aforementioned control systems, especially the lateral fronto-parietal subsystem. Together, we conclude that similar to what we emphasized in the temporal domain, ongoing activity variations also show a nested structure in the spatial domain that expresses an embedding of modularity into a hierarchy.

\section{THE FUNCTION OF ONGOING BRAIN ACTIVITY FUNCTIONAL CONNECTIVITY, STRUCTURAL CONNECTIVITY AND COGNITIVE CONTEXT}

The difficulties in adequately capturing the spatiotemporal form of intrinsic brain activity that we have discussed in the previous section should not be thought of as mere empty battles of nomenclature. This form is important when pondering the function of intrinsic activity, and any proposal with respect to this function will be benchmarked against its potential for accounting for this spatiotemporal structure. The perspective that we have proposed in the previous section for functional connectivity is reminiscent of descriptions of structural brain connectivity and we have already appealed to these similarities in the tree metaphor (Bullmore and Sporns, 2009). A hypothesis about what determines the form of ongoing brain activity that ensues is that intrinsic functional connectivity simply reflects some neural "noise" that plays out on a non-random structural connectivity; and therefore takes on the shape of a limited set of spatial patterns (i.e., dynamics on structure). Indeed, computational simulations of functional connectivity using noisy input generate functional covariance patterns that reflect underlying structural circuitry (Sporns et al., 2000). And empirical evidence has been reported showing strong correspondence of intrinsic functional and anatomical connectivity (Skudlarski et al., 2008; Greicius et al., 2009). In more comprehensive investigations, at the level of the entire brain, this match has been confirmed but systematic quantitative analysis also revealed that it is not perfect. In other words, structural connectivity permitted only a partial prediction of the empirically observed functional connectivity (Honey et al., 2009). Of course, the imperfection in predicting functional from structural connectivity could simply reflect limitations in the methods applied for data acquisition and analysis. Yet, an important alternative hypothesis is that with underlying structural connectivity as a backbone functional connectivity is shaped by additional context-dependent modulation.

At first glance, this hypothesis seems to be at odds with the persistence of spatial ICN patterns across different levels of context and consciousness, from task- and stimulus-induced active states (Fair et al., 2007; Golland et al., 2007; Eckert et al., 2008), over resting wakefulness (Greicius et al., 2003; Fox et al., 2005; Fransson, 2005), light and deep sleep (Horovitz et al., 2007, 2009; Nir et al., 2008), light sedation (Greicius et al., 2008), to deep anesthesia in monkeys (Vincent et al.,2007) and severe disorders of consciousness as in vegetative state patients (Boly et al., 2009). Furthermore, the finding of robust intrinsic activity patterns in the absence of consciousness also suggests that intrinsic activity fluctuations cannot be considered merely or entirely the neural correlates of conscious, mentation or mind-wandering that in the absence of an explicit task paradigm simply remains experimentally uncontrolled (Buckner and Vincent, 2007).

However, evidence in favor of the hypothesis that ongoing brain activity is in fact context-sensitive has now been accumulated by a range of studies. Although functional connectivity patterns persist qualitatively across wide ranges of different functional contexts, as mentioned above, they do nonetheless express quantitative 
changes. They differ for instance quantitatively between the healthy awake brain and the brain in a state of pathological unconsciousness, where functional connectivity within the so-called defaultmode network decreases with the degree of consciousness; across minimally conscious state, vegetative state and ultimately coma (Vanhaudenhuyse et al., 2010). They also differ quantitatively in the healthy brain between wakefulness and deep (slow-wave) sleep, a state of physiological unconsciousness (Horovitz et al., 2009). It is noteworthy that the reduction in connectivity between posterior and frontal areas of the default-mode network during sleep is anatomically selective, and that fluctuation amplitudes within regions remain unchanged. This result makes it unlikely that modulations in intrinsic connectivity simply reflect a change of noise levels propagating through an anatomically connected system.

And even during the state of wakefulness (and on a shorter time scale) intrinsic connectivity patterns express differences that can be related to recent cognitive experience. Over the course of one scanning session, i.e., a time span that in all likelihood does not involve gross structural connectivity changes, adaptive modulation of intrinsic functional connectivity has been reported after visuomotor learning (Albert et al., 2009), episodic memory (Tambini et al., 2010) and language tasks (Waites et al., 2005; Hasson et al., 2009). These findings show that functional context interacts with the expression of intrinsic activity and thus motivates further experimental investigation of the functional significance of intrinsic activity.

A common critique of these latter studies is that they might collapse "true intrinsic" activity with reverberating traces of previous cognitive experience. Of course, the same critique holds for "pure" resting-state studies during wakefulness, because they at least include task-unrelated mind-wandering that constitutes an ongoing cognitive content (Mason et al., 2007; Christoff et al., 2009) and by its very nature cannot be considered to lack context. The only way to dissociate "true intrinsic" activity from more specifically context-related neural processes would be if there were spatiotemporal hallmarks selectively tagging intrinsic activity. Our review of its temporal and spatial form, however, suggests, at least to us, that no such properties can currently be identified with confidence. Alternatively, one may question whether such dissociation is inevitably justified and necessary and this leads one to consider the actual function of ongoing activity. We propose that its function is intimately related to cognition, and this relation is inherent to the brain, be it in a "resting" or active state. This proposal could seem at odds with the studies that we have discussed above and that show qualitative spatial correspondence between ICNs across very different functional brain states. But it is as true that ICNs strongly resemble spatial patterns with sets of regions that typically co-activate (or deactivate) in cognitive activation studies as a function of the paradigm (Smith et al., 2009). We therefore argue that function cannot be assigned purely on the basis of spatial patterns.

\section{COGNITIVE CONSEQUENCES OF SPONTANEOUS ACTIVITY FLUCTUATIONS}

In this section, we review a different way of addressing the function of ongoing activity. In this approach, the functional consequences of ongoing activity are assessed by studying whether fluctuations in ongoing activity can account for behavioral variability. From the previous sections we can derive the following predictions for such an account: (1) ongoing activity should affect behavior with a time constant that is sufficiently slow to be captured by hemodynamic signals. (2) The spatial pattern within which ongoing activity affects behavior should be context-dependent and should be detectable precisely at that position within a hierarchical structure that best matches the functional demands of a given context. In the following, we review evidence for both predictions from recent neuroimaging studies.

Two lines of earlier research suggested that there might indeed be a link between ongoing activity fluctuations and behavioral variability. One is that behavioral performance when repeating the same task over and over again shows fluctuations with a qualitatively similar temporal profile as ongoing activity, i.e., high power at low frequencies (Gilden, 2001). The other is that neural responses evoked by identical stimuli fluctuate over time. The latter effect has been very explicitly tied to ongoing activity fluctuations by examining the dependence of evoked response variations on trialby-trial fluctuations of pre-stimulus activity levels. In an influential study, Arieli et al. (1996) investigated ongoing and stimulus-evoked activity with concurrent optical and electrophysiological methods in anesthetized cats. They found that variability of evoked responses could be largely accounted for by the initial level of ongoing activity just prior to stimulus onset. Their data show a linear relationship between ongoing activity immediately before stimulation and evoked activity levels. Simply adding the averaged stimulusrelated activity increment to the pattern of ongoing activity in an individual trial provided an excellent prediction of the actually measured activity level during the evoked response in that trial. Recently, several functional neuroimaging studies have not only revisited this issue but also established links between neural and behavioral variability.

\section{Functional imaging findings}

At a very different spatial and temporal resolution than Arieli et al., Fox et al. (2006a) made a similar observation using fMRI. They found that trial-to-trial variability of finger movementrelated activity in motor cortex could be largely accounted for by ongoing activity fluctuations measured in the contralateral motor cortex, the one ipsilateral to the finger that was moved (Figure 1). Their clever approach tackled the problem that the relative contribution of ongoing and task-related activity cannot be separated by analyzing activity in the task-relevant region during the evoked response. By removing trial by trial the simultaneously recorded activity level in a region that belongs to the same ICN but was not engaged by the task from the signal in the task-relevant region they "cleaned away" the ongoing and retained the evoked component.

From the perspective of data analysis in functional imaging, this procedure is very attractive. It suggests that averaging across trials provides a good way for estimating a veridical evoked activity change, the response, and that the latter shows little if any variability. And removing the variability related to ongoing activity and hence tightening the residual variability of the evoked response estimate yields a clear-cut gain in statistical sensitivity. Yet, the same group established in a subsequent study that the trial-by-trial variability 


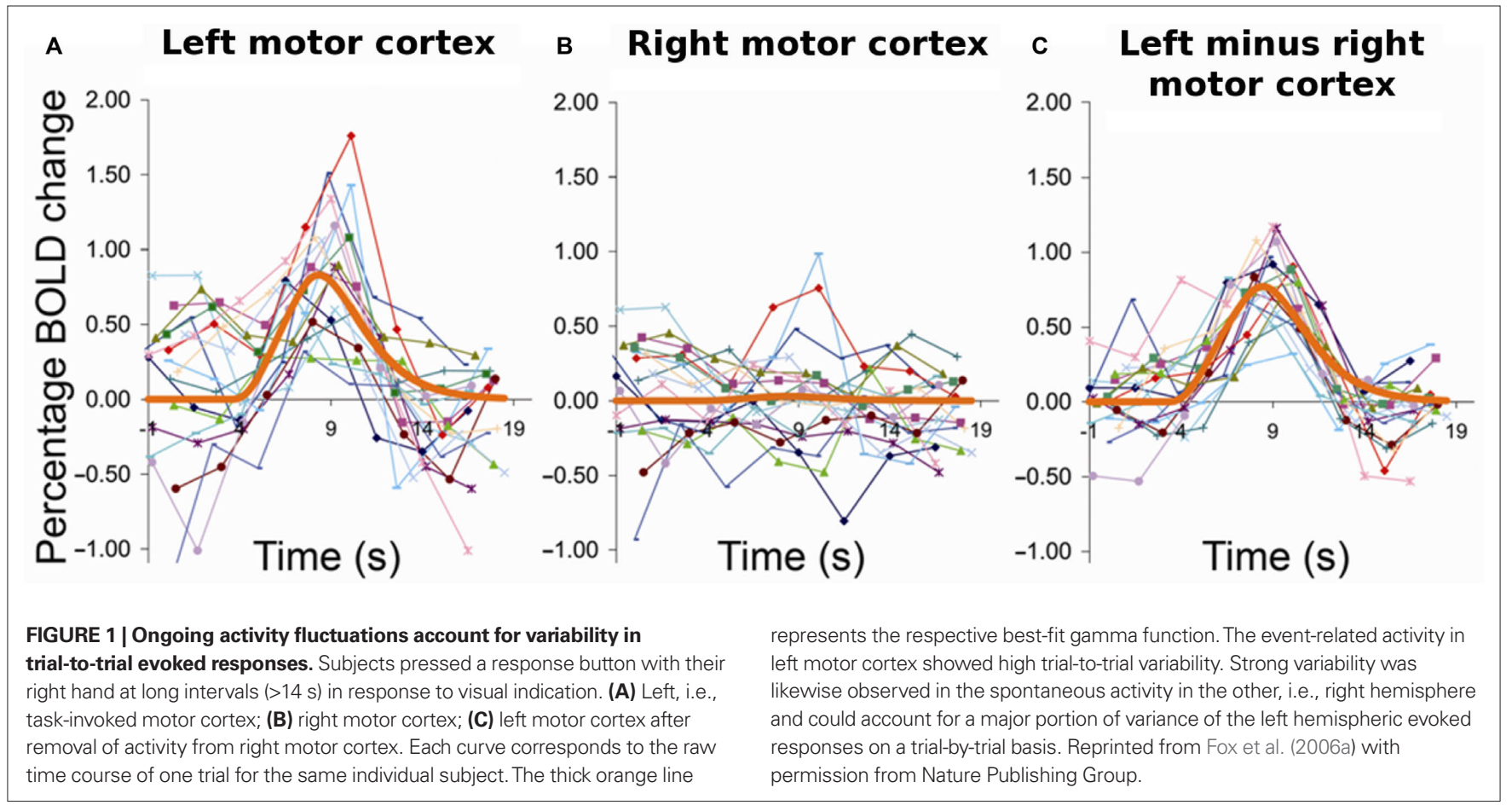

in task-related motor cortex activation was functionally meaningful and translated into behavioral variability as measured by the force that subjects applied in different trials when pressing a response button (Fox et al., 2007). In other words, the aforementioned procedure of removing inter-trial variability, albeit attractive from a signal processing perspective, is far less tempting for that line of research which seeks to establish neural correlates of behavior.

From a methodological point of view, a limitation of the aforementioned approach is that it is grounded in the assumption that motor cortex ipsilateral to the moving finger is silent in this paradigm. Indeed, distal upper limb movements are represented almost exclusively contralaterally but with greater force they involve coinnervation of more proximal musculature, which in turn is represented more bilaterally in motor cortex (reviewed in Kleinschmidt and Toni, 2004). Other groups have therefore explored alternative approaches to the issue of whether ongoing activity fluctuations are functionally relevant. Instead of using simultaneously recorded signal in a region that belongs to the same ICN but is silent in a task context, several groups have taken pre-stimulus signal in the same region that will subsequently respond to a given stimulus as a measure of ongoing activity. This approach is hence similar to the one adopted by Arieli et al. (1996) but on a different time scale.

Boly et al. (2007) investigated the perceptual impact of prestimulus activity fluctuations in a somatosensory detection task. For somatosensory stimuli close to perceptual threshold pre-stimulus activity levels in large distributed systems resembling ICNs indicated whether or not a stimulus was perceived on a given trial. The system biasing towards perceiving the stimulus comprised the thalamus, dorsal anterior cingulate cortex (dACC) and anterior insula/inferior frontal gyrus, as well as parieto-frontal areas including intraparietal sulcus and dorso-lateral prefrontal cortex. As discussed in the previous section, these areas commonly show activation or "task-positive" behavior in a wide range of cognitive task settings (Corbetta et al., 2002; Smith et al., 2009). Conversely, on trials where subjects missed the threshold stimulus, pre-stimulus activity levels were higher in posterior cingulate (PCC), parahippocampal and lateral parietal components of the default-mode network. This latter network is known to show deactivation or "task-negative" behavior in most task settings (Gusnard and Raichle, 2001).

Taken together, these observations could further support a simple dichotomy in which higher ongoing activity in "task-positive" brain networks would facilitate perceptual performance whereas higher activity levels in the default-mode network would degrade performance. A recent study speaks against the generality of this scenario by showing that functional context determines in which brain regions ongoing activity will affect perceptual performance and whether this will be a facilitating or detrimental effect (Sadaghiani et al., 2009). In a free-response, auditory detection task, we presented broad-band noise stimuli in unpredictable intervals of 20-40 s and at individual detection threshold. Subjects pressed a button whenever they perceived the target sound. Successful detection as compared to misses was preceded by significantly higher pre-stimulus activity in early auditory cortex (Figure $\mathbf{3 A}$ ) as well as in two ICNs. Perceptual performance was better with higher pre-stimulus activity in a network comprising thalamus, anterior insula and dACC, which suggests a role for this ICN in maintaining alertness and task-set (Figure 2A). Conversely, and counter to common intuition, higher baseline activity in the dorsal attention system of parietal and frontal areas biased towards misses (Figure 2B) presumably expressing the lack of spatial connotation in our stimulus and task. The observation of opposite effects in these two task-positive ICNs shows that in spite of shared variance, the networks are sufficiently segregated to exert independent influences on perceptual outcome. And finally, higher baseline activity in 


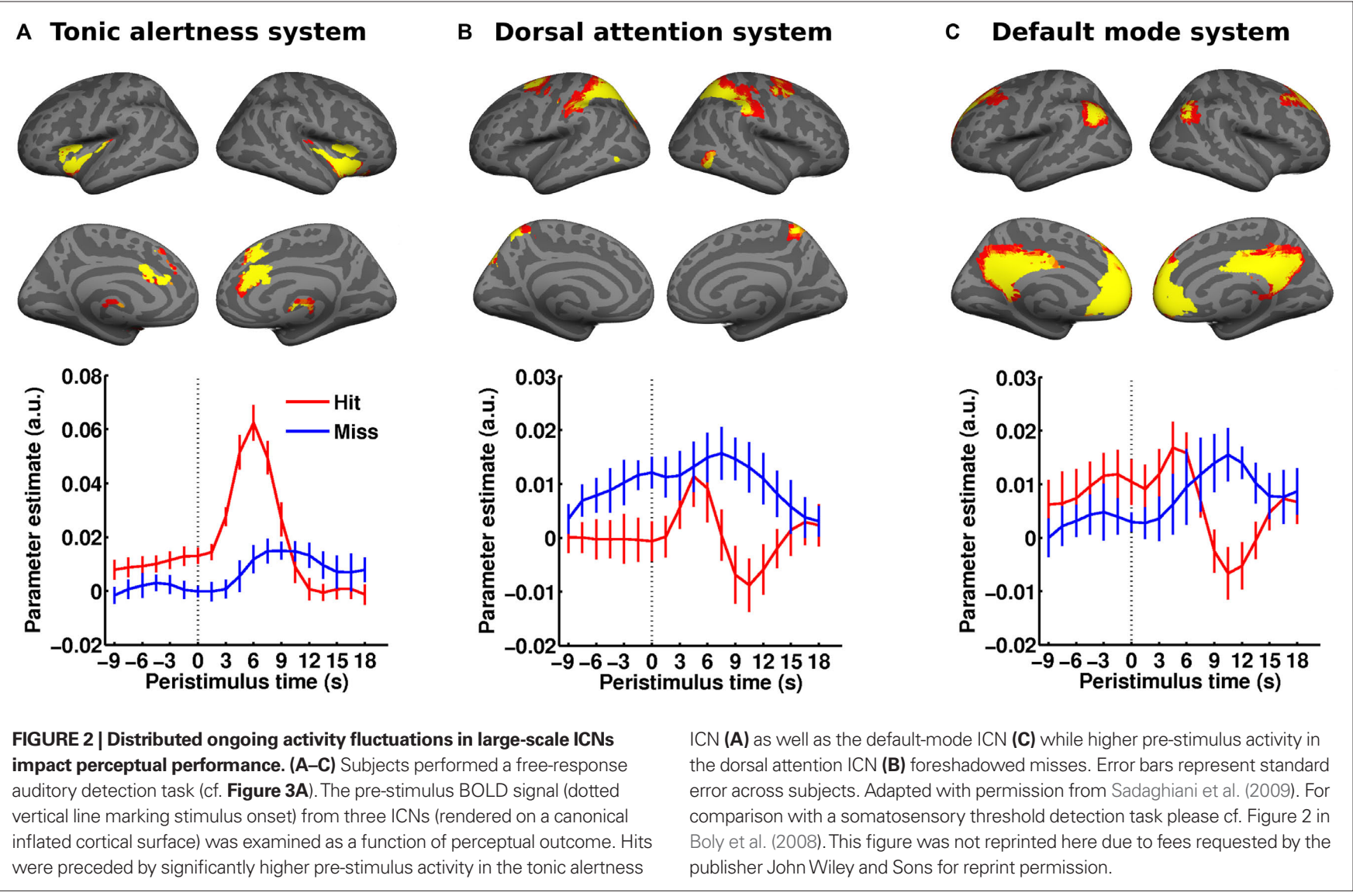

the precuneus/PCC region of the default-mode network preceded hits, which in turn yielded a biphasic response with a "task-positive" activation component preceding the typical but delayed deactivation (Figure 2C). At first glance, this finding might appear at odds with the existing literature but it probably reflects the importance of retrieving a memory template of the target for successful performance on the continuous sensory input (Shannon and Buckner, 2004; Daselaar et al., 2009).

Thus, in the context of a non-localized and non-semantic auditory stimulus and a task that depends on recognition memory but not spatial attention, the usual effects from activity in default-mode and dorsal spatial attention systems were reversed. Of note, the time courses of pre-stimulus effects in these two networks were very distinct, making it unlikely that signal change in one was simply (epiphenomenally) mirrored by that in the other. In other words, these opposite effects were presumably independent of one another rather than reflecting a hard-wired antagonism between these two ICNs that others have claimed based on the observation of intrinsic anticorrelation (Fox et al., 2005). These findings highlight that context determines the influence ongoing fluctuations exert on stimulus processing and ultimately perception.

It seems fair to posit that where and how ongoing activity fluctuations impact on perceptual decisions depends on which sensory features and cognitive faculties are relevant in a given context. Accordingly, one might expect that in perceptual decisions, which do not involve an all-or-none success of detection but a choice between two closely matched alternatives, generic contributions from ICNs become less important and that a pre-stimulus effect might only be detectable in a single task-relevant region (rather than throughout the entire network to which this region belongs). In this case, it would be purely local variations in activity and not those throughout a distributed system that would exert an influence (cf. previous section on hierarchical structure of ongoing activity). Such a mechanism would make it mandatory to estimate ongoing activity from pre-stimulus signal in the task-relevant region instead of from simultaneous signal in a reference region of the same ICN. Evidence for such a scenario has been provided by two separate but closely related experiments.

In a perceptual decision task on Rubin's ambiguous vase-faces figure, subjects had to report on each trial whether they perceived the vase or the two faces in profile. The presentation of the stimuli was sparse at long and variable intervals (range 20-50 s), and the stimuli were presented only briefly (Hesselmann et al., 2008b). Subjects reported face percepts on approximately half of the trials and vase percepts on the other trials. Higher pre-stimulus activity levels in the right fusiform face area (FFA), a region specialized for face processing, were found to bias towards the percept of faces rather than a vase (Figure 3B). This finding was later replicated in the domain of visual motion perception (Hesselmann et al., 2008a). In this study, short events of random dot motion with near-threshold coherence levels were presented, and subjects indicated on each trial whether they perceived coherent or random motion. Here, subjects' perceptual decisions were biased by 


\section{A Auditory detection}
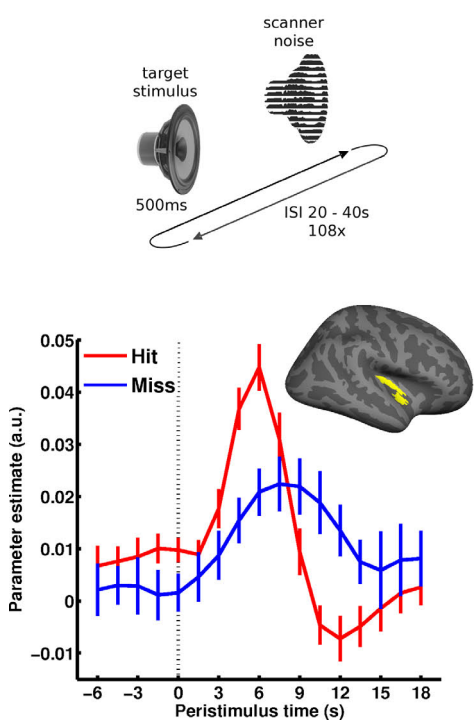

B Faces/vase decision
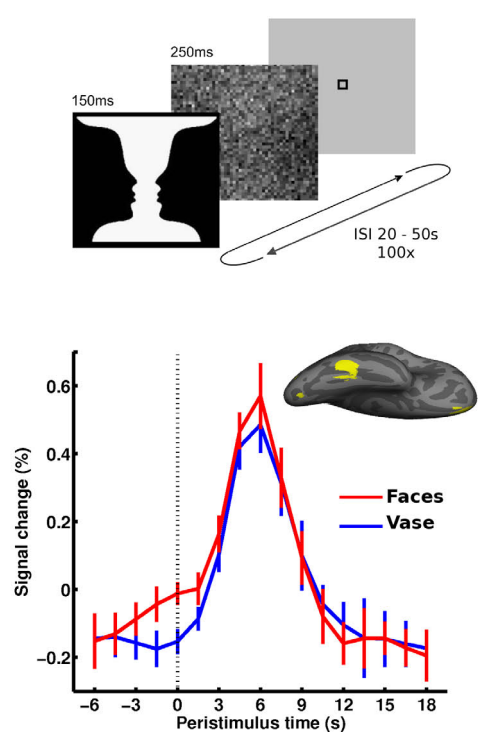

c Motion decision

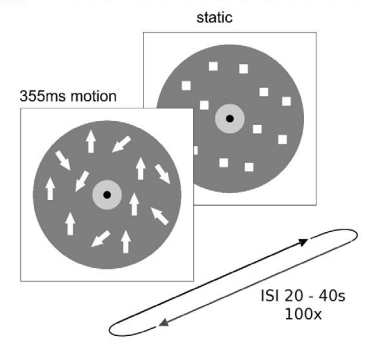

FIGURE 3 | Local spontaneous variations in ongoing activity of specialized sensory regions impact perception. The upper part illustrates the paradigm: (A) auditory detection experiment: in a free-response setting subjects detected an auditory target stimulus presented at perceptual threshold. (B) Perceptual decision on an ambiguous figure: subjects reported either faces or vase perception in response to flashes of the faces-vase ambiguous figure.

(C) Motion decision experiment: random dot motion was presented at motion coherence threshold and subjects decided trial by trial whether motion was coherent or random. In all experiments, trials followed at long and unpredictable intervals. In each experiment, the pre-stimulus BOLD signal (dotted vertical line marking stimulus onset) was examined as a function of perceptual outcome and sampled from accordingly specialized sensory areas. The corresponding regions of interest (early auditory cortex, FFA and hMT+, respectively) are presented on a canonical inflated cortical surface of the right hemisphere. In all experiments, higher pre-stimulus time course in the respective sensory region biased towards perceiving stimulus properties for which these regions are particularly sensitive. Error bars represent standard error across subjects. For more details see Hesselmann et al. (2008a,b); Sadaghiani et al. (2009). pre-stimulus activity levels in right middle temporal cortex (V5/ $\mathrm{hMT}+$ ), a region crucially involved in the analysis and perception of wide-field coherent motion. Specifically, perception of coherent motion was preceded by significantly higher ongoing activity in V5/hMT+ (Figure 3C). In both experiments, no other task-related cortical regions showed a significant link between pre-stimulus activity and perceptual outcome.

\section{Electro- and magnetoencephalographic findings}

Electro- (EEG) and magnetoencephalography (MEG) studies have also established links between ongoing activity and behavior. While less informative in terms of spatial localization, these studies have identified distinct oscillation bands that carry signals, which predict perceptual performance. Using MEG, Jensen and colleagues observed that visual discriminability of a threshold stimulus decreased with an increase in pre-stimulus occipito-parietal $\alpha$ band power (van Dijk et al., 2008). Likewise, they reported that in a go no-go task false alarms were preceded by higher levels of $\alpha$ band power in the occipital cortex and bilateral somatosensory cortices ( $\mu$ rhythm) as compared to correct withholds on no-go trials (Mazaheri et al., 2009). Not only responses to natural stimuli but also to artificial direct cortical stimulation are influenced by the power of ongoing oscillations: Using transcranial magnetic stimulation, phosphene-perception was only induced following lower pre-stimulation $\alpha$ amplitudes (Romei et al., 2008), suggesting that occipital alpha power indexes cortical excitability. Evidence link- ing such observations in EEG frequency bands to the infra-slow frequency range covered by imaging studies comes from work by Palva and colleagues. They investigated pre-stimulus power fluctuations using full-band EEG sensitive to infra-slow fluctuations $(<0.1 \mathrm{~Hz})$ in a somatosensory threshold detection task within a free-response setting. They found highest detection rates and shortest reaction times to be associated with intermediate power levels (inverse $u$-shaped relation) of $\alpha, \beta$ and $\gamma$ band oscillations over sensorimotor cortices, and with highest power of these bands over parietal electrodes (Linkenkaer-Hansen et al., 2004). Interestingly, in this task setting the phase of infra-slow fluctuations was found to be strongly correlated to the power of higher frequencies $(1-40 \mathrm{~Hz})$ and to be highly predictive of hits and misses on a trial-by-trial basis (Monto et al., 2008). Recently, electrophysiological studies have not only shown power but also phase of band-limited oscillatory activity to affect perceptual performance. For example, trial-to-trial variability in perceptual outcome has been related to the phase of EEG $\alpha$ and $\theta$ band oscillations in visual threshold detection tasks (Busch et al., 2009; Mathewson et al., 2009).

\section{THE RELATION OF ONGOING AND EVOKED NEURAL ACTIVITY}

The above findings are important because they show that across many temporal scales variability in ongoing activity - which is commonly obscured by normalization to pre-stimulus baseline-contributes to the way in which the brain (and ultimately, the observer) responds to sensory stimuli. The imaging studies show that the topography of 
these effects is compatible with a hierarchical view on intrinsic brain activity and depends on context. In the two experiments discussed above, which involve fairly subtle perceptual decisions, we targeted areas that we considered likely to respond more strongly during one of the two possible perceptual interpretations of the ambiguous stimuli used. Despite identical sensory input in each experiment, we indeed confirmed that face-percept trials using the Rubin stimulus yielded higher evoked FFA responses and coherent-percept trials using the dot motion stimulus higher evoked hMT+ responses (Figures 3B,C). Together with the aforementioned effects observed in pre-stimulus signal these findings could be believed to confirm a behavior equivalent to the one in the study by Arieli et al. (1996) that we discussed above. In other words, a single stimulus would, on each trial, evoke a fixed activity increment which would add to the level of ongoing activity encountered on that trial. Variations in ongoing activity would then determine perceptual outcome by yielding variations in peak activity that would, or not, pass a threshold required for a perceptual decision. By such a mechanism, even a simple additive relationship between ongoing and evoked activity could become functionally significant (note that we have to call on a threshold mechanism - which is by definition non-linear - to make a linear effect of ongoing activity functionally interesting).

The important consequence from such a mechanism - that also provides an easily testable hypothesis - would be that the relation between ongoing and evoked activity should not depend on perceptual outcome because the latter would be determined solely by the peak activity of the response. We could reject this hypothesis in both experiments by showing a significant interaction between evoked and ongoing activity when predicting perceptual outcome. Specifically, peak and pre-stimulus activity levels in hMT+ correlated less when dot motion was perceived as coherent rather than random (Figures 4A,B). Likewise, peak activity levels in FFA were significantly less correlated with pre-stimulus signal when subjects perceived faces than when they reported a vase (Figures 4C,D). These observations show that the mechanism by which ongoing activity affects subsequent perception is independent from the one that can be observed during stimulus processing. In other words, the latter does not result from a mere passive propagation of effects preceding stimulus presentation. The theoretical implications of these findings for models of perceptual decision-making have been discussed in the respective publications (Hesselmann et al., 2008a,b). Yet, there is reason to believe that both linear (e.g., under anesthesia, Arieli et al., 1996, or in passive viewing, Bianciardi et al., 2009) and non-linear interactions can be observed and future work will need to clarify which parameters determine the regime under which ongoing and evoked activity interact (see Kisley and Gerstein, 1999, for a study on changes in linearity as a function of depth of anesthesia).

\section{THE NATURE OF ONGOING BRAIN ACTIVITY Comparing spontaneous fluctuations and variability after cueing}

We have argued above that ongoing activity is modulated by cognitive context and that spontaneous activity fluctuations can be thought of as fluctuations of an internal and predictive contextual representation. It therefore appears sensible to compare results from such studies with those where context has been explicitly modulated by introducing cues that prepare for an upcoming cognitive challenge. Several studies have employed such cues to study the variability of evoked neural and perceptual responses to a single stimulus (or a group of equivalent stimuli) (Super et al., 2003; Sapir et al., 2005; Thut et al., 2006; Wyart and Tallon-Baudry, 2009). Typically, in these paradigms, a cue will appear that can indicate a task-relevant location or feature for a stimulus that will be presented after a brief but often variable delay. These studies in general try to understand which neural mechanisms underpin selective attention. As behavior and evoked responses are modulated by attention, variability in a cue-induced anticipatory signal that correlates with perceptual performance on the subsequent stimulus can be considered a neural signature of preparatory attention. Of course, this interpretation does not speak to the mechanism that generates this variability in the first place but only suggests that attentional preparation is subject to a variability of an unknown origin that is behaviorally relevant. The similarity of this conclusion with that from the aforementioned studies on spontaneous fluctuations suggests a need for closer examination and comparison.

From our perspective, variability in cortical activity following an orientating cue presents a special case and currently remains ambiguous. One interpretation could be that this variability is the same as that seen in ongoing activity and that the cue will hence be more or less efficient, both neurally and perceptually, as a function of the state of the system prior to cueing. Another view could be that the neural response elicited by the cue could in itself be variable and that this variability translates into perceptual performance. As we have discussed previously, simply removing the effects of pre-cue baseline would not permit arbitrating between these two scenarios, since the amplitude of the cue response may be subject to interactions with pre-cue activity levels. However, analyses as those reviewed above that preserve pre-cue "baseline" signal fluctuations could be used to disambiguate the functional nature of cued settings.

Another line of comparison regards the interpretation of the cortical signal. If the pre-stimulus signal expresses a level of preparatory attention in studies using cues, does this permit the conclusion that in studies without cues fluctuations of ongoing activity can be thought of as fluctuations in attention? If one were to make this claim it would have to survive a couple of benchmark checks. The most important one is that evoked responses to target stimuli should be enhanced by attention. This enhancement could reflect anything between a true response gain as implied in earlier studies (Chawla et al., 1999) and a simple additive effect of fixed stimulus-driven increment in the presence of an increased background activity (Sylvester et al., 2009). In both our studies addressing signal variations in the absence of cues, however, the opposite behavior was found. The higher pre-stimulus signal was, the smaller the actual incremental evoked response amplitude in regions that were critical to the percept on those trials, i.e., V5/ $\mathrm{hMT}+$ for motion coherence detection (Figures 4A,B) and the FFA for face perception (Figures 4C,D).

\section{Predictive coding and free-energy formulations}

So how can these observations about intrinsic fluctuations be understood functionally? We will address this under a predictive coding account of neuronal activity, given that cues furnish exogenous and explicit predictions. In what follows, it is important to realize that optimal predictions or expectations rest on two distinct processes. The first is predicting the content of a percept (e.g., what caused 


\section{Trial-by-trial correlation of ongoing and evoked activity}
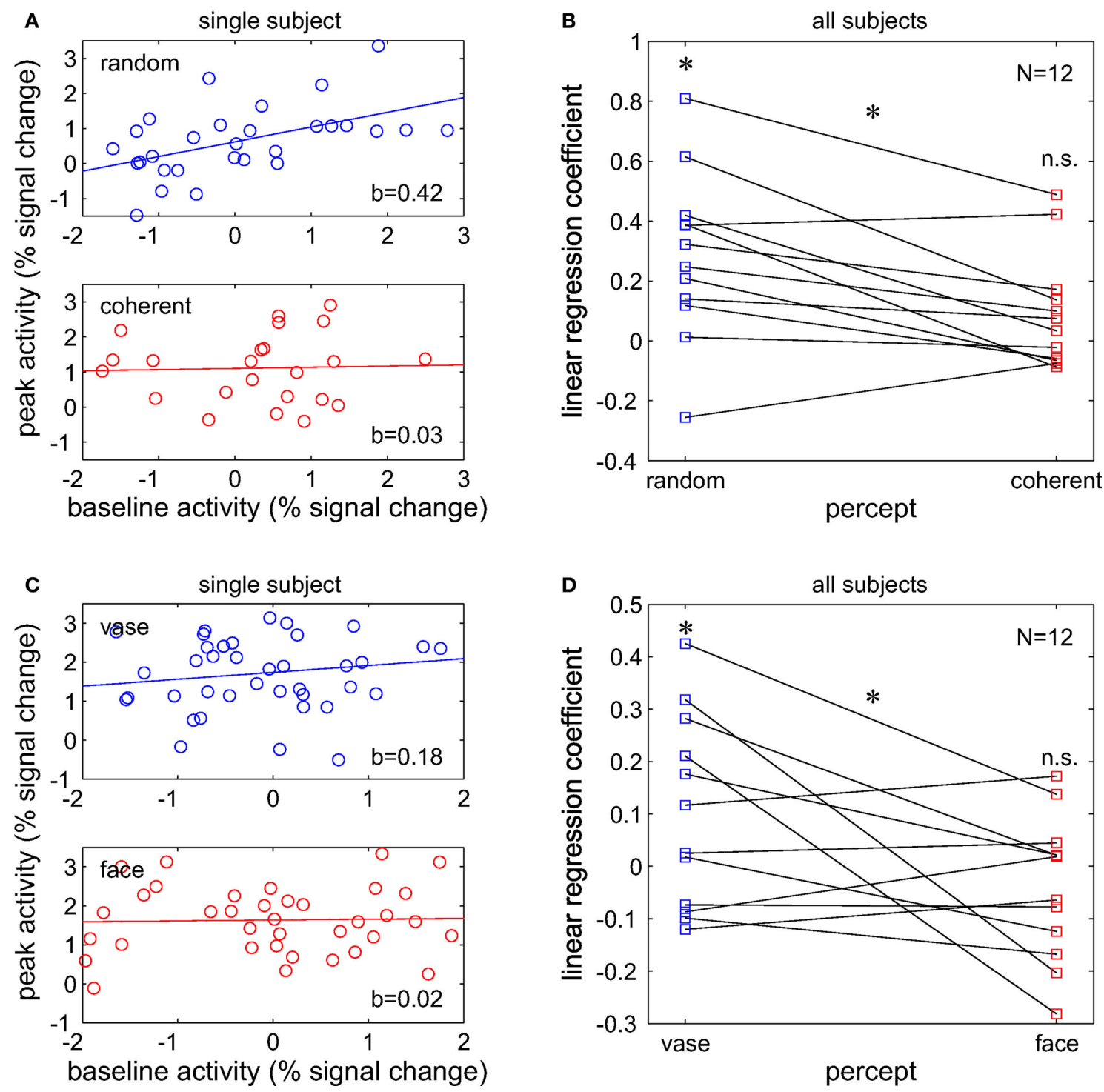

FIGURE 4 | Percept-dependent and non-linear interaction of evoked responses with pre-stimulus baseline activity. Percept-dependent linear regression was performed between trial-by-trial pre-stimulus activity at $-1.5 \mathrm{~s}$ and peak activity at $6 \mathrm{~s}$ relative to stimulus onset. For two independent experiments, the regression is illustrated for one representative single subject and the regression coefficient is given for the group. (A,B) The motion decision experiment (for stimuli cf. Figure $\mathbf{3 C}$ ): coefficients were significantly larger than 0 when motion was perceived as coherent $\left(t_{11}=3.55, p<0.01\right)$ but not when it was perceived as random ( $t_{11}=1.7$, n.s.) and significantly different between the two perceptual outcomes ( $t_{11}=3.24, p<0.01$, paired). Adapted with permission from Hesselmann et al. (2008a). (C,D) The face-vase decision experiment (cf.

Figure 3B): likewise, coefficients showed a trend $>0$ when faces were perceived $\left(t_{11}=1.88, p=0.087\right)$ but not when the vase was perceived $\left(t_{11}=-1.06\right.$, n.s.). Importantly, they were significantly different between the two perceptual outcomes ( $t_{11}=2.31, p<0.05$, paired). Dataset from Hesselmann et al. (2008b). All tests are two-sided t statistics. the stimulus) and the second is properly inferring the uncertainty or precision of that prediction (e.g., the probabilistic context in which a stimulus appears). This difference is illustrated nicely by the difference between the effects of cueing and priming.

Cues are usually employed in attentional paradigms to guide predictions about task-relevant locations or features (context) but not about the actual target (content). In other words, knowing that a target will appear at a given location within the next couple of seconds does not provide any information about the content of the target's features; e.g., whether a grating will be slanted to the left or right. Cues call for allocation of attentional resources to the appropriate sensory channels, without biasing to one outcome in these channels, or another. In what follows, we consider this in terms of optimizing the synaptic gain of selected channels. This may also help understand the electrophysiological correlates of nonspatial attentional or perceptual processes; e.g., related to the feature 
class, in contrast to spatial attention (Wyart and Tallon-Baudry, 2009). Conversely, sensory priming induces expectations about the content of sensory input, which we will assume is mediated by priming-dependent changes in synaptic activity and efficacy. In accord with this view, priming effects are associated with reduced evoked response amplitudes and are, of course, readily embraced by predictive coding accounts (Henson, 2003).

Recently, it has been proposed that a single fundamental principle might govern brain activity underlying action, perception, attention and learning (Friston, 2005, 2009, 2010). In its most simple form, the free-energy principle states that the brain seeks to minimize surprise (more formally, the negative log-probability of a sensory outcome). This is achieved by continuously updating an internal model that generates top-down predictions of sensory input. Unexpected sensory inputs that cannot be "explained away" by an internal model of the current states of the world emerge as bottom-up prediction errors (hence predictive coding). These prediction errors are accumulated or assimilated by higher cortical areas to update the model and optimize its predictions. Perception rests on the optimization of top-down predictions (or, model) to best explain away the bottom-up prediction error caused by incoming sensory information, a notion embraced by Bayesian formulations (Kersten et al., 2004; Hohwy et al., 2008). In the present context, the free-energy formulation is of interest because it covers many observations about evoked responses but it is not confined to them. When applied to the specific issue of ongoing cortical activity and its relation to evoked responses (and subsequent perception), the free-energy principle can account for many reported empirical findings and yields further testable predictions.

The free-energy formulation (Friston, 2009) requires the brain to represent the causes of sensory input (by optimizing synaptic activity; i.e., perceptual inference), and its internal model of contextual and causal regularities (by optimizing short and long-term changes in synaptic gain and efficacy; i.e, attention and peceptual learning). Crucially, all changes in synaptic activity, gain and efficacy minimize the same thing; namely free energy, which under some simplifying assumptions is just the amount of prediction error. In line with this view, Lewis et al. (2009) observed that intensive training shapes intrinsic connectivity between visual areas and higher order frontal and parietal regions that presumably generate visuospatial top-down predictions. In terms of the distinction above, synaptic (neuronal) activity encodes the content percepts, while synaptic gain encodes contextual precision (cf. attentional gain). In what follows, we will consider ongoing activity as reflecting neuronal activity that predicts the causes of sensory inputs and then turn to interpretations that cover fluctuations in synaptic gain or precision.

\section{Ongoing activity and predictions}

Perceptual inference and learning speaks to a general principle, according to which past experiences inform predictions of the future to optimize behavior. The idea that ongoing activity patterns reflect a historically informed internal model of causal dynamics in the world (that serves to generate predictions of future sensory input) fits nicely with the role of neural "replay" in memory formation (Jeffery, 2004; Foster and Wilson, 2006). Indeed, the itinerant (wandering or searching) dynamics that characterize intrinsic fluctuations have been proposed as mathematical models of short-term memory (Bick and Rabinovich, 2009) and have been discussed explicitly in terms of free-energy minimization (Kiebel et al., 2009b).

It is important to realize that this interpretation does not restrict the role of ongoing activity to brain states that are accessible to introspection. The most basic version of this mechanism might be seen during the perception of music and speech, where, mathematically, the itinerant dynamics conform to stable heteroclinic channels that show winner-less competition (Seliger et al., 2003; Kiebel et al., 2009a). However, these dynamics also manifest in the absence of sensory information - just because sensory inputs are not currently available does not mean that the brain models the world as having stopped. Important examples here include optimization (consolidation) of synaptic strengths during sleep (Vyazovskiy et al., 2008; Diekelmann and Born, 2010). Another example is optimization or selection of competing internal models, using itinerant searches over different hypotheses (models) about the world. This view links itinerant (wandering) dynamics to "mind wandering" often invoked to explain resting-state fluctuations. This link provides a formal and precise role for ongoing itinerant activity that has been exploited in perception (e.g., Kiebel et al., 2009a) and planning (e.g., Namikawa and Tani, 2010). In machine learning and robotics, the itinerancy mandated by sensitivity to initial conditions and some forms of chaotic dynamics is now one of the main candidates for explaining how trajectories into the future are explored and selected. This fits comfortably with the notion that brain activity can be formulated in terms of itinerant dynamics (e.g., Tsuda, 2001). One important feature of itinerancy is that it enables ongoing activity to express fluctuations that ensure transitions between different (meta)stable neuronal states (Deco et al., 2009). Itinerant fluctuations of this activity reflect the dynamic nature of the underlying internal model that does not remain locked in a stationary mode but remains malleable by continuously exploring hypotheses regarding future experience and action. It is for this reason that functional connectivity measures, which describe the extent of wandering activity (and not stationary activity levels), provide such an informative description. Similar neural population behavior has also been observed on shorter temporal and smaller spatial scales (Wackermann et al., 1993; Kenet et al., 2003).

\section{Ongoing activity and precision}

In free-energy formulations of predictive coding, a major contributor to measured neuronal activity is precision-weighted prediction error. This precision weighting is implemented by increases in synaptic gain (cf. attentional modulation) so that prediction errors are boosted selectively according to the context established by predictions or cues). This means that fluctuating activity levels may reflect not just itinerant optimization of predictions but fluctuations in their precision. Evidence for this interpretation of ongoing activity fluctuations (as a modulation in precision or gain afforded to afferent information) comes from investigations of false vs. correct perceptual inference. Intrinsic brain activity (as indexed by fMRI signal) could be interpreted as a correlate of sensory evidence in random walk or race models (in essence an extension of signal detection theory over time Smith and Ratcliff, 2004; Gold and Shadlen, 2007) or as a proxy for precision in free-energy formulations of predictive coding (Friston, 
2008). Crucially, these two accounts can be tested against findings in threshold detection paradigms discussed above (Hesselmann et al., 2008a; Sadaghiani et al., 2009). The former (evidence accumulation) framework suggests high pre-stimulus activity (i.e., a high starting level for the random walk) will bias towards subsequent stimulus detection (true hits or false alarms). Conversely, the latter (predictive coding) framework suggests that high ongoing activity (i.e., precise prediction errors) will bias towards subsequently correct inference (hits or correct rejections). In two independent datasets, we recently found that pre-stimulus activity levels were associated with the latter perceptual outcome and hence support the interpretation of ongoing activity as reflecting the precision of perceptual inference (Hesselmann et al., 2010) (Figure 5).

The implementation of precision in the predictive coding framework is necessitated by the presence of noise in environmental states or sensory input and plays a key role in regulating the reliability or relative weighting of bottom-up prediction errors against top-down predictions. Thus, this gain could represent a mechanism that is very suitable for mediating selective attention (Friston, 2009). Of note however, a shared final common neural pathway does not imply that fluctuations in ongoing activity necessarily reflect fluctuations in attention (cf. the discussion of cueing paradigms in the previous section and itinerant optimization of neuronal activity above). In conclusion, the free-energy formulation presents an attractive theoretical framework for a unified approach to a diversity of neurophysiological observations, including those related to ongoing activity fluctuations.

\section{SUMMARY}

In the recent years, intrinsic brain activity has become a new and enticing focus of interest and research into brain function (Fox and Raichle, 2007). In spite of conceptual concerns about studying unconstrained brain activity (Morcom and Fletcher, 2007a,b) studies of intrinsic brain activity during rest as well as in paradigm settings have proven very fruitful in understanding the functional role of ongoing activity and its relation to cognitive processes (Buckner et al., 2008; Greicius, 2008; Hesselmann et al., 2008b; Sadaghiani et al., 2009).

Ongoing activity is organized in a functional architecture at various temporal and spatial scales (Kenet et al., 2003; Bassett et al., 2006; Meunier et al., 2009). It has been established that evoked neural responses are embedded into this underlying functional architecture (Tsodyks et al., 1999) and cannot be fully understood in isolation from the context established by ongoing activity. Therefore, trial-to-trial variability in evoked responses is not just noise but a non-random function of network fluctuations (Fontanini and Katz, 2008). For this reason the current review of ongoing activity considered its spatiotemporal structure in relation to moment-to-moment variability in cognition.
A

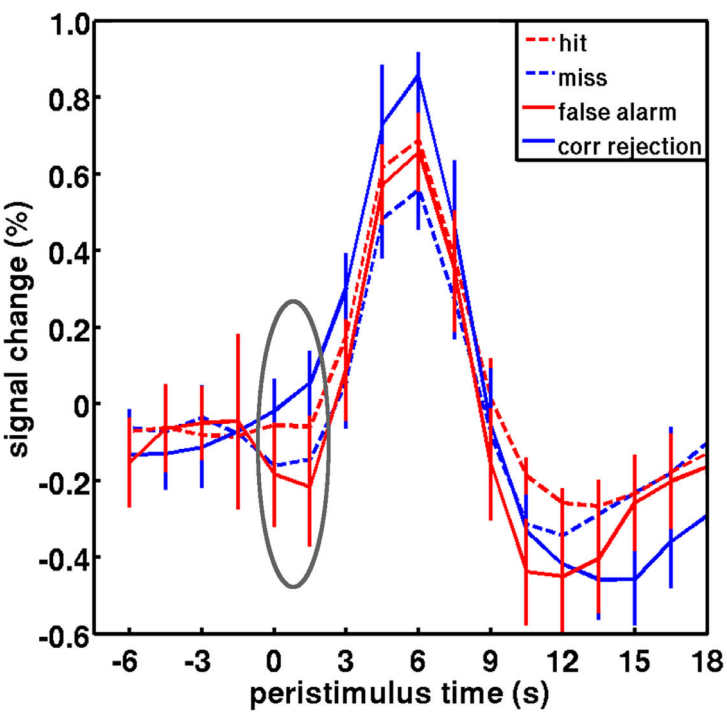

FIGURE 5 | Baseline activity levels in false vs. correct inferences are captured by the predictive coding framework. (A) Peristimulus fMRI signal time courses from the motion decision experiment: for stimuli and region of interest cf. Figure $\mathbf{3 C}$. Hits and misses correspond to trials at threshold motion coherence level (on average 13\%), while correct rejections and false alarms correspond to occasional trails with a quasi-random coherence level (1\%). Pre-stimulus activity showed a main effect of accuracy, correct vs. incorrect (consistent with predictive coding), but no main effect of percept, coherent vs. incoherent (predicted by evidence accumulation). Pre-stimulus activity prior to hits was significantly greater than misses; and pre-stimulus activity in false alarms were significantly less than in correct rejects. (B) Peristimulus time courses from the auditory detection experiment: for stimuli and region of

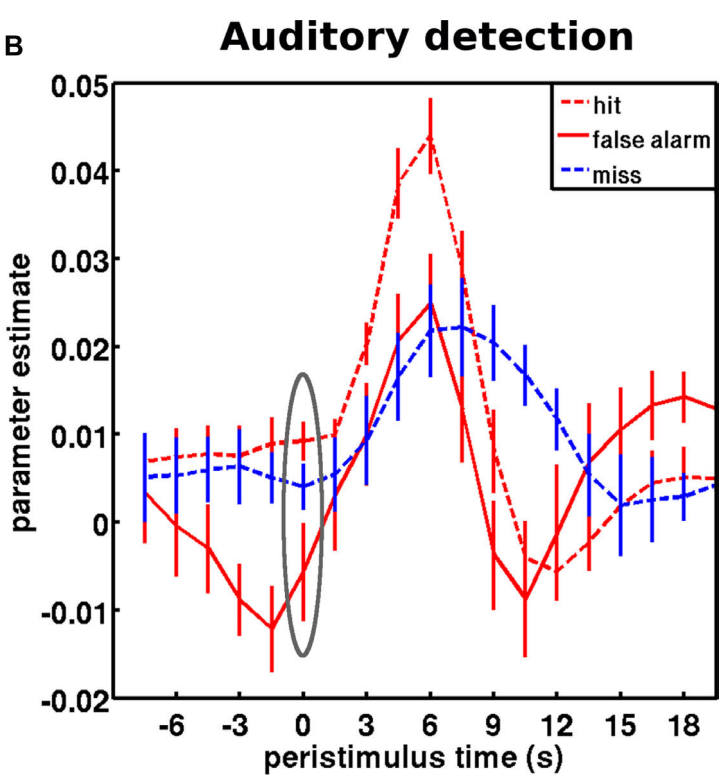

interest cf. Figure 3A. False alarms occurred occasionally when subjects reported to hear the target stimulus in the absence of stimulation. As assumed by predictive coding, false inference (false alarms and misses) were each preceded by significantly lower levels of activity in auditory cortex than veridical hits. Note that this free-response paradigm does not furnish correct rejection trials (i.e., subjects are not required to indicate the stimulus is absent). The gray ellipse covers the pre-stimulus period submitted to statistical testing. The time courses for hits and misses correspond to the respective time courses in Figure 3. However, note that only a subset of subjects that had a sufficient number of wrong inferences was included in this analysis. Error bars represent standard error across subjects. Adapted with permission from Hesselmann et al. (2010). 
With respect to structure, we emphasized that behaviorally relevant ongoing activity is hierarchically organized and does not seem restricted to clear-cut temporal or spatial scales. The spatial patterns of ICNs and the membership of constituent regions are gradual and display a global-to-local connectivity, reminiscent of small-world topologies (Bullmore and Sporns, 2009). We further discussed that the strength of these correlations is constrained by structural connectivity but is modulated by mental states and current context, strongly suggesting a functional component to intrinsic activity fluctuations (i.e., dynamics on structure).

We have tried to substantiate the role of intrinsic fluctuations in terms of the necessarily itinerant dynamics entailed by internal (generative) models of the world the brain might use to make predictions about its sensorium. In doing this, we hoped to establish a formal link between the notion of mind wandering and itinerancy (wandering dynamics) in computational accounts of perceptual learning and inference. Furthermore, we extended this account to include the modulation of prediction error signals by their precision and suggested that measured fluctuations in neuronal activity may reflect modulations in synaptic gain; of the sort seen in fast synchronized neuronal exchanges and attentional modulation.

\section{REFERENCES}

Albert, N. B., Robertson, E. M., and Miall, R. C. (2009). The resting human brain and motor learning. Curr. Biol. 19, 1023-1027.

Arieli, A., Sterkin, A., Grinvald, A., and Aertsen, A. (1996). Dynamics of ongoing activity: explanation of the large variability in evoked cortical responses. Science 273, 1868-1871.

Bassett, D. S., Meyer-Lindenberg, A., Achard, S., Duke, T., and Bullmore, E. (2006). Adaptive reconfiguration of fractal small-world human brain functional networks. Proc. Natl. Acad. Sci. U.S.A. 103, 19518-19523.

Beckmann, C. F., DeLuca, M., Devlin, J. T., and Smith, S.M. (2005). Investigations into resting-state connectivity using independent component analysis. Philos. Trans. R. Soc. Lond., B, Biol. Sci. 360, 1001-1013.

Bianciardi, M., Fukunaga, M., van Gelderen, P., Horovitz, S. G., de Zwart, J. A., and Duyn, J. H. (2009). Modulation of spontaneous fMRI activity in human visual cortex by behavioral state. Neuroimage 45, 160-168.

Bick, C., and Rabinovich, M. I. (2009). Dynamical origin of the effective storage capacity in the brain's working memory. Phys. Rev. Lett. 103, 218101.

Biswal, B. B., Kylen, J. V., and Hyde, J. S. (1997). Simultaneous assessment of flow and BOLD signals in resting-state functional connectivity maps. NMR. Biomed. 10, 165-170.
Boly, M., Balteau, E., Schnakers, C., Degueldre, C., Moonen, G., Luxen, A., Phillips, C., Peigneux, P., Maquet, P., and Laureys, S. (2007). Baseline brain activity fluctuations predict somatosensory perception in humans. Proc. Natl. Acad. Sci. U.S.A. 104, 12187-12192.

Boly,M., Phillips, C., Balteau, E., Schnakers, C., Degueldre, C., Moonen, G., Luxen, A., Peigneux, P., Faymonville, M.-E., Maquet, P., and Laureys, S. (2008). Consciousness and cerebral baseline activity fluctuations. Hum. Brain Mapp. 29, 868-874.

Boly, M., Tshibanda, L., Vanhaudenhuyse, A., Noirhomme, Q., Schnakers, C., Ledoux, D., Boveroux, P., Garweg, C., Lambermont, B., Phillips, C., Luxen,A., Moonen, G., Bassetti, C., Maquet, P., and Laureys, S. (2009). Functional connectivity in the default network during resting state is preserved in a vegetative but not in a brain dead patient. Hum. Brain Mapp. 30, 2393-2400.

Buckner, R. L., Andrews-Hanna, J. R., and Schacter, D. L. (2008). The brain's default network: anatomy, function, and relevance to disease. Ann. N. Y. Acad. Sci. 1124, 1-38.

Buckner, R. L., and Vincent, J. L. (2007). Unrest at rest: default activity and spontaneous network correlations. Neuroimage 37, 1091-1096.

Bullmore, E., and Sporns, O. (2009). Complex brain networks: graph theoretical analysis of structural and functional systems. Nat. Rev. Neurosci. 10, 186-198.

It has been argued that evoked response variability is coordinated rather than arbitrary and substantiates the very essence of cognition as it translates into behavioral variability (Buzsáki, 2006). From this point of view, we reviewed evidence that ongoing brain activity fluctuations influence trial-by-trial behavior and perception of identical stimuli. Site and sign of this impact depend on cognitive context and the functional role of a given region or neuroanatomical system. This cognitive variability over time is the visible manifestation of a continuous trajectory of ongoing activity, an itinerant trajectory that can be thought of as a memory trace building up within a complex and adaptive system. Intrinsic activity hence constitutes the brain's internal context for processing external information and generating behavior (Kenet et al., 2003; Fontanini and Katz, 2008). Future progress on these issues can only be made by treating ongoing activity as a signal instead of noise and by shifting from a behaviorist stance that records evoked responses to a constructivist approach that interrogates neural history records as the substrate of a process of active perceptual synthesis.

\section{ACKNOWLEDGMENTS}

S. Sadaghiani is supported by the Friedrich-Ebert Foundation (Germany). G. Hesselmann is supported by a Minerva fellowship (Max Planck Society). Our research is funded by the Agence Nationale de la Recherche (SPONTACT grant), France.

Busch, N. A., Dubois, J., and VanRullen, R. (2009). The phase of ongoing EEG oscillations predicts visual perception. J. Neurosci. 29, 7869-7876.

Buzsáki, G. (2006). Rhythms of the Brain New York: Oxford UP.

Chawla, D., Rees, G., and Friston, K. J. (1999). The physiological basis of attentional modulation in extrastriate visual areas. Nat. Neurosci. 2 , 671-676.

Christoff, K., Gordon, A. M., Smallwood, J., Smith, R., and Schooler, J.W. (2009). Experience sampling during fMRI reveals default network and executive system contributions to mind wandering. Proc. Natl. Acad. Sci. U.S.A. 106, 8719-8724.

Cohen, A. L., Fair, D. A., Dosenbach, N. U. F., Miezin, F. M., Dierker, D., Van Essen, D. C., Schlaggar, B. L., and Petersen, S. E. (2008). Defining functional areas in individual human brains using resting functional connectivity MRI. Neuroimage 41, 45-57.

Cole, D., Smith, S., and Beckamnn, C. (2010). Advances and pitfalls in the analysis and interpretation of restingstate FMRI data. Front. Syst. Neurosci. 4:8. doi: 10.3389/fnsys.2010.00008.

Corbetta, M., Kincade, J.M., and Shulman, G. L. (2002). Neural systems for visual orienting and their relationships to spatial working memory. J. Cogn. Neurosci. 14, 508-523.

Corbetta, M., and Shulman, G. L. (2002) Control of goal-directed and stimulusdriven attention in the brain. Nat. Rev Neurosci. 3, 201-215.
Daselaar, S. M., Prince, S. E., Dennis, N. A., Hayes, S. M., Kim, H., and Cabeza, R. (2009). Posterior midline and ventral parietal activity is associated with retrieval success and encoding failure. Front. Hum. Neurosci. 3:13. doi: 10.3389/neuro.09.013.2009.

Deco, G., Rolls, E. T., and Romo, R. (2009). Stochastic dynamics as a principle of brain function. Prog. Neurobiol. 88, 1-16.

Diekelmann, S., and Born, J. (2010). The memory function of sleep. Nat. Rev. Neurosci. 11, 114-126.

Dosenbach, N. U. F., Fair, D. A., Miezin, F. M., Cohen, A. L., Wenger, K. K., Dosenbach, R. A. T., Fox, M. D., Snyder, A. Z., Vincent, J. L., Raichle, M. E., Schlaggar, B. L., and Petersen, S. E. (2007). Distinct brain networks for adaptive and stable task control in humans. Proc. Natl. Acad. Sci. U.S.A. 104, 11073-11078.

Dosenbach, N. U. F., Visscher, K. M., Palmer, E. D., Miezin, F. M., Wenger, K. K., Kang, H. C., Burgund, E. D., Grimes, A. L., Schlaggar, B. L., and Petersen, S. E. (2006). A core system for the implementation of task sets. Neuron 50, 799-812.

Eckert, M. A., Menon, V., Walczak, A., Ahlstrom, J., Denslow, S., Horwitz, A., and Dubno, J. R. (2008). At the heart of the ventral attention system: the right anterior insula. Hum. Brain Mapp. 30, 2530-2541.

Fair, D. A., Schlaggar, B. L., Cohen, A. L., Miezin, F. M., Dosenbach, N. U. F., Wenger, K. K., Fox, M. D., Snyder, A. 
Z., Raichle, M. E., and Petersen, S. E. (2007). A method for using blocked and event-related fMRI data to study "resting state" functional connectivity. Neuroimage 35, 396-405.

Ferrarini, L., Veer, I. M., Baerends, E., van Tol, M. J., Renken, R. J., van der Wee, N. J., Veltman, D. J., Aleman, A., Zitman, F. G., Penninx, B. W., van Buchem, M. A., Reiber, J. H., Rombouts, S. A., and Milles, J. (2009). Hierarchical functional modularity in the resting-state human brain. Hum. Brain Mapp. 30, 2220-2231.

Fontanini, A., and Katz, D. B. (2008). Behavioral states, network states, and sensory response variability. J. Neurophysiol. 100, 1160-1168.

Foster, D. J., and Wilson, M. A. (2006). Reverse replay of behavioural sequences in hippocampal place cells during the awake state. Nature 440, 680-683.

Fox, M. D., and Raichle, M. E. (2007). Spontaneous fluctuations in brain activity observed with functional magnetic resonance imaging. Nat. Rev. Neurosci. 8, 700-711.

Fox, M. D., Snyder, A. Z., Vincent, J. L., Corbetta, M., Van Essen, D. C., and Raichle, M. E. (2005). The human brain is intrinsically organized into dynamic, anticorrelated functional networks. Proc. Natl. Acad. Sci. U.S.A. 102, 9673-9678.

Fox, M. D., Snyder, A. Z., Vincent, J. L., and Raichle, M.E. (2007). Intrinsic fluctuations within cortical systems account for intertrial variability in human behavior. Neuron 56, 171-184.

Fox, M. D., Snyder, A. Z., Zacks, J. M., and Raichle, M.E. (2006a).Coherent spontaneous activity accounts for trial-totrial variability in human evoked brain responses. Nat. Neurosci. 9, 23-25.

Fox, M. D., Corbetta, M., Snyder, A. Z., Vincent, J. L., and Raichle, M. E. (2006b). Spontaneous neuronal activity distinguishes human dorsal and ventral attention systems. Proc. Natl. Acad. Sci. U.S.A. 103, 10046-10051.

Fox, M. D., Zhang, D., Snyder, A. Z., and Raichle, M.E. (2009). The global signal and observed anticorrelated resting state brain networks. J. Neurophysiol. 101, 3270-3283.

Fransson, P. (2005). Spontaneous lowfrequency BOLD signal fluctuations: an fMRI investigation of the restingstate default mode of brain function hypothesis. Hum. Brain Mapp. 26, 15-29.

Freeman, W. J., Rogers, L. J., Holmes, M. D., and Silbergeld, D. L. (2000). Spatial spectral analysis of human electrocorticograms including the alpha and gamma bands. J. Neurosci. Methods 95, 111-121.
Friston, K. (2005). A theory of cortical responses. Philos. Trans. R. Soc. Lond., B, Biol. Sci. 360, 815-836.

Friston, K. (2008). Hierarchical models in the brain. PLoS Comput. Biol. 4, e1000211. doi: 10.1371/journal. pcbi. 1000211 .

Friston, K. (2009). The free-energy principle: a rough guide to the brain? Trends Cogn. Sci. 13, 293-301.

Friston, K. (2010). The free-energy principle: a unified brain theory? Nat. Rev Neurosci. 11, 127-138.

Gilden, D. L. (2001). Cognitive emissions of 1/f noise. Psychol. Rev. 108, 33-56.

Gold, J. I., and Shadlen, M. N. (2007). The neural basis of decision making. Annu. Rev. Neurosci. 30, 535-574.

Golland, Y., Bentin, S., Gelbard, H. Benjamini, Y., Heller, R., Nir,Y., Hasson, U., and Malach, R. (2007). Extrinsic and intrinsic systems in the posterior cortex of the human brain revealed during natural Sensory Stimulation. Cereb. Cortex 17, 766-777.

Golland, Y., Golland, P., Bentin, S., and Malach, R. (2008). Data-driven clustering reveals a fundamental subdivision of the human cortex into two global systems. Neuropsychologia 46, 540-553.

Greicius, M. (2008). Resting-state functional connectivity in neuropsychiatric disorders. Curr. Opin. Neurol. 21, 424-430.

Greicius, M. D., Kiviniemi, V., Tervonen, O., Vainionpää, V., Alahuhta, S., Reiss, A. L., and Menon, V. (2008). Persistent default-mode network connectivity during light sedation. Hum. Brain Mapp. 29, 839-847.

Greicius, M.D., Krasnow, B., Reiss, A.L., and Menon,V.(2003). Functional connectivity in the resting brain: a network analysis of the default mode hypothesis. Proc. Natl. Acad. Sci. U.S.A. 100, 253-258.

Greicius, M. D., Supekar, K., Menon, V., and Dougherty, R. F. (2009). Restingstate functional connectivity reflects structural connectivity in the default mode network. Cereb. Cortex (New York, NY: 1991) 19, 72-78.

Gusnard, D. A., and Raichle, M.E. (2001) Searching for a baseline: functional imaging and the resting human brain. Nat. Rev. Neurosci. 2, 685-694.

Hasson, U., Nusbaum, H. C., and Small, S. L. (2009). Task-dependent organization of brain regions active during rest. Proc. Natl. Acad. Sci. U.S.A. 106, 10841-10846.

He, B. J., Snyder, A. Z., Zempel, J. M. Smyth, M. D., and Raichle, M. E. (2008).Electrophysiological correlates of the brain's intrinsic large-scale functional architecture. Proc. Natl. Acad. Sci. U.S.A. 105, 16039-16044.

He, B. J., Zempel, J. M., Snyder, A. Z., and Raichle, M. E. (2010). The temporal structures and functional significance of scale-free brain activity. Neuron 66 353-369.

Henson, R. N. (2003). Neuroimaging studies of priming. Prog. Neurobiol. 70, 53-81.

Hesselmann, G., Kell, C. A., and Kleinschmidt, A. (2008a). Ongoing activity fluctuations in hMT+ bias the perception of coherent visual motion. J. Neurosci. 28, 14481-14485.

Hesselmann, G., Kell, C. A., Eger, E. and Kleinschmidt, A. (2008b). Spontaneous local variations in ongoing neural activity bias perceptual decisions. Proc. Natl. Acad. Sci. U.S.A. 105, 10984-10989.

Hesselmann, G., Sadaghiani, S., Friston, K. J., and Kleinschmidt, A. (2010) Predictive coding or evidence accumulation? False inference and neuronal fluctuations. PLoS ONE 5, e9926. doi: 10.1371/journal.pone.0009926.

Hohwy, J., Roepstorff, A., and Friston K. (2008). Predictive coding explains binocular rivalry: an epistemological review. Cognition 108, 687-701.

Honey, C. J., Sporns, O., Cammoun, L. Gigandet, X., Thiran, J. P., Meuli, R. and Hagmann, P. (2009). Predicting human resting-state functional connectivity from structural connectivity. Proc. Natl. Acad. Sci. U.S.A. 106 2035-2040.

Horovitz, S. G., Braun, A. R., Carr, W. S., Picchioni, D., Balkin, T. J., Fukunaga, M., and Duyn, J.H. (2009). Decoupling of the brain's default mode network during deep sleep.Proc. Natl. Acad. Sci. U.S.A. 106, 11376-11381.

Horovitz, S. G., Fukunaga, M., de Zwart, J. A., van Gelderen, P., Fulton, S. C. Balkin, T. J., and Duyn, J. H. (2007) Low frequency BOLD fluctuations during resting wakefulness and light sleep: a simultaneous EEG-fMRI study. Hum. Brain Mapp. 29, 671-682.

Jeffery, K. J. (2004). Remembrance of futures past. Trends Cogn. Sci. (Regul. Ed.) 8, 197-199.

Kenet, T., Bibitchkov, D., Tsodyks, M. Grinvald, A., and Arieli, A. (2003). Spontaneously emerging cortical representations of visual attributes. Nature 425, 954-956.

Kersten, D., Mamassian, P., and Yuille, A (2004). Object perception as Bayesian inference. Annu. Rev. Psychol. 55, 271-304.

Kiebel, S. J., Daunizeau, J., and Friston, K. J. (2009a). Perception and hierarchical dynamics.Front. Neuroinformatics 3:20 doi: 10.3389/neuro.11.020.2009.

Kiebel, S. J., von Kriegstein, K., Daunizeau, J., and Friston, K. J. (2009b). Recognizing sequences of sequences. PLoS Comput. Biol. 5, e1000464. doi: 10.1371/journal.pcbi.1000464.

Kisley, M. A., and Gerstein, G. L. (1999). Trial-to-trial variability and state-dependent modulation of auditory-evoked responses in cortex. $J$. Neurosci. 19, 10451-10460.

Kleinschmidt, A., and Toni, I. (2004) "Functional magnetic resonance imaging of the human motor cortex," in Motor Cortex in Voluntary Movements: A Distributed System for Distributed Functions, eds A. Riehle and E. Vaadia, Boca-Raton, FL: CRC Press, pp. 49-84.

Laufs, H., Krakow, K., Sterzer, P., Eger, E., Beyerle, A., Salek-Haddadi, A., and Kleinschmidt, A. (2003). Electroencephalographic signatures of attentional and cognitive default modes in spontaneous brain activity fluctuations at rest. Proc. Natl. Acad. Sci. U.S.A. 100, 11053-11058.

Leopold, D. A., Murayama, Y., and Logothetis, N. K. (2003). Very slow activity fluctuations in monkey visual cortex: implications for functional brain imaging. Cereb. Cortex 13, 422-433.

Lewis, C. M., Baldassarre,A., Committeri, G., Romani, G. L., and Corbetta, M. (2009). Learning sculpts the spontaneous activity of the resting human brain. Proc. Natl. Acad. Sci. U.S.A. 106, 17558-17563.

Linkenkaer-Hansen, K., Nikouline, V. V., Palva, J. M., and Ilmoniemi, R. J. (2001). Long-range temporal correlations and scaling behavior in human brain oscillations. J. Neurosci. 21, 1370-1377.

Linkenkaer-Hansen, K., Nikulin, V. V. Palva, S., Ilmoniemi, R. J., and Palva, J. M. (2004). Prestimulus oscillations enhance psychophysical performance in humans. J. Neurosci. 24 10186-10190.

Mandelbrot, B. (1998). Multifractals and 1/f Noise: Wild Self-Affinity in Physics. New York: Springer

Margulies, D. S., Kelly, A. M. C., Uddin, L. Q., Biswal, B. B., Castellanos, F. X., and Milham, M. P. (2007). Mapping the functional connectivity of anterior cingulate cortex. Neuroimage 37, 579-588.

Margulies, D. S., Vincent, J. L., Kelly, C., Lohmann, G., Uddin, L. Q., Biswal, B. B., Villringer, A., Castellanos, F. X., Milham, M. P., and Petrides, M. (2009). Precuneus shares intrinsic functional architecture in humans and monkeys. Proc. Natl. Acad. Sci. U.S.A 106, 20069-20074.

Marrelec, G., Bellec, P., Krainik, A., Duffau, H., Pelegrini-Issac, M. Lehericy, S., Benali, H., and Doyon, J. (2008). Regions, systems, and the brain: hierarchical measures of functional integration in fMRI. Med. Image Anal. 12, 484-496.

Mason, M. F., Norton, M. I., Van Horn, J. D., Wegner, D. M., Grafton, S. T., and Macrae, C. N. (2007). Wandering 
minds: the default network and stimulus-independent thought. Science 315, 393-395.

Mathewson, K. E., Gratton, G., Fabiani, M., Beck, D. M., and Ro, T. (2009). To see or not to see: prestimulus \{alpha\} phase predicts visual awareness. $J$. Neurosci. 29, 2725-2732.

Mazaheri,A., Nieuwenhuis, I. L., van Dijk, H., and Jensen, O. (2009). Prestimulus alpha and mu activity predicts failure to inhibit motor responses. Hum. Brain Mapp. 30, 1791-1800.

McDonnell, M. D., and Abbott, D. (2009). What is stochastic resonance? Definitions, misconceptions, debates, and its relevance to biology. PLoS Comput. Biol. 5, e1000348. doi: 10.1371/journal.pcbi.1000348.

Meunier, D., Lambiotte, R., Fornito, A., Ersche, K. D., and Bullmore, E. T. (2009). Hierarchical modularity in human brain functional networks. Front. Neuroinformatics 3:37. doi: 10.3389/neuro.11.037.2009

Monto, S., Palva, S., Voipio, J., and Palva, J. M. (2008). Very slow EEG fluctuations predict the dynamics of stimulus detection and oscillation amplitudes in humans. J. Neurosci. 28, 8268-8272.

Morcom, A. M., and Fletcher, P. C. (2007a). Does the brain have a baseline? Why we should be resisting a rest. Neuroimage 37, 1073-1082.

Morcom, A. M., and Fletcher, P. C. (2007b). Cognitive neuroscience: the case for design rather than default. Neuroimage 37, 1097-1099.

Murphy, K., Birn, R. M., Handwerker, D. A., Jones, T. B., and Bandettini, P. A. (2009). The impact of global signal regression on resting state correlations: are anti-correlated networks introduced? Neuroimage 44, 893-905.

Namikawa, J., and Tani, J. (2010). Learning to imitate stochastic time series in a compositional way by chaos. Neural Netw. 23, 625-638.

Nir, Y., Fisch, L., Mukamel, R., GelbardSagiv, H., Arieli, A., Fried, I., and Malach, R. (2007). Coupling between neuronal firing rate, gamma LFP, and BOLD fMRI is related to interneuronal correlations. Curr. Biol. 17, 1275-1285.

Nir, Y., Mukamel, R., Dinstein, I., Privman, E., Harel, M., Fisch, L., Gelbard-Sagiv, H., Kipervasser, S., Andelman, F., Neufeld, M. Y., Kramer, U., Arieli, A., Fried, I., and Malach, R. (2008). Interhemispheric correlations of slow spontaneous neuronal fluctuations revealed in human sensory cortex. Nat. Neurosci. 11, 1100-1108.

Raichle, M. E. (2010). Two views of brain function. Trends Cogn. Sci. (Regul.Ed.) 14, 180-190.

Romei, V., Brodbeck, V., Michel, C., Amedi, A., Pascual-Leone, A., and
Thut, G. (2008). Spontaneous fluctuations in posterior alpha-band EEG activity reflect variability in excitability of human visual areas. Cereb. Cortex 18, 2010-2018.

Sadaghiani, S., Hesselmann, G., and Kleinschmidt, A. (2009). Distributed and antagonistic contributions of ongoing activity fluctuations to auditory stimulus detection. J. Neurosci. 29, 13410-13417.

Sapir, A., d'Avossa, G., McAvoy, M., Shulman, G. L., and Corbetta, M. (2005). Brain signals for spatial attention predict performance in a motion discrimination task. Proc. Natl. Acad. Sci. U.S.A. 102, 17810-17815.

Schölvinck, M. L., Maiera, A., Yec, F. Q., Duynd, J. H., and Leopold, D. A. (2010). Neural basis of global restingstate fMRI activity. Proc. Natl. Acad. Sci. U.S.A. 107, 10238-10243.

Seeley, W. W., Menon, V., Schatzberg, A. F., Keller, J., Glover, G. H., Kenna, H., Reiss, A. L., and Greicius, M. D. (2007). Dissociable intrinsic connectivity networks for salience processing and executive control. J. Neurosci. 27, 2349-2356.

Seliger, P., Tsimring, L. S., and Rabinovich, M. I. (2003).Dynamics-based sequential memory: winnerless competition of patterns. Phys. Rev. E. Stat. Nonlin. Soft Matter Phys. 67, 011905.

Shannon, B. J., and Buckner, R. L. (2004). Functional-anatomic correlates of memory retrieval that suggest nontraditional processing roles for multiple distinct regions within posterior parietal cortex. J. Neurosci. 24, 10084-10092.

Shmuel, A., and Leopold, D. A. (2008). Neuronal correlates of spontaneous fluctuations in fMRI signals in monkey visual cortex: implications for functional connectivity at rest. Hum. Brain Mapp. 29, 751-761.

Skudlarski, P., Jagannathan, K., Calhoun, V.D., Hampson, M., Skudlarska, B. A., and Pearlson, G. (2008). Measuring brain connectivity: diffusion tensor imaging validates resting state temporal correlations. Neuroimage 43 , 554-561.

Smith, P. L., and Ratcliff, R. (2004) Psychology and neurobiology of simple decisions. Trends Neurosci. 27, 161-168.

Smith, S. M., Fox, P. T., Miller, K. L., Glahn, D. C., Fox, P. M., Mackay, C. E., Filippini, N., Watkins, K. E., Toro, R., Laird, A. R., and Beckmann, C. F. (2009). Correspondence of the brain's functional architecture during activation and rest. Proc. Natl. Acad. Sci. U.S.A. 106, 13040-13045.

Sporns, O., Tononi, G., and Edelman, G. M. (2000). Theoretical neuroanatomy: relating anatomical and functional connectivity in graphs and cortical connection matrices. Cereb. Cortex 10, 127-141.

Sridharan, D., Levitin, D. J., and Menon, V. (2008). A critical role for the right fronto-insular cortex in switching between central-executive and defaultmode networks. Proc. Natl. Acad. Sci. U.S.A. 105, 12569-12574.

Sterzer, P., and Kleinschmidt, A. (2010) Anterior insula activations in perceptual paradigms-often observed but barely understood. Brain Struct. Funct. 10.1007/s00429-010-0252-2. [Epub ahead of print].

Super, H., van der Togt, C., Spekreijse, H. and Lamme, V. A. F. (2003). Internal state of monkey primary visual cortex (V1) predicts figure-ground perception. J. Neurosci. 23, 3407-3414.

Sylvester, C. M., Shulman, G. L., Jack, A. I. and Corbetta, M. (2009). Anticipatory and stimulus-evoked blood oxygenation level-dependent modulations related to spatial attention reflect a common additive signal. J. Neurosci. 29, 10671-10682.

Tambini,A., Ketz,N., and Davachi,L. (2010). Enhanced brain correlations during rest are related to memory for recent experiences. Neuron 65, 280-290.

Thut, G., Nietzel, A., Brandt, S. A., and Pascual-Leone, A. (2006). Alpha-band electroencephalographic activity over occipital cortex indexes visuospatia attention bias and predicts visual target detection. J. Neurosci. 26, 9494-9502.

Tsodyks, M., Kenet, T., Grinvald, A., and Arieli, A. (1999). Linking spontaneous activity of single cortical neurons and the underlying functional architecture. Science 286, 1943-1946.

Tsuda, I. (2001). Toward an interpretation of dynamic neural activity in terms of chaotic dynamical systems. Behav. Brain Sci. 24, 793-810

van Dijk, H., Schoffelen, J.-M., Oostenveld, R., and Jensen, O. (2008). Prestimulus oscillatory activity in the alpha band predicts visual discrimination ability. J. Neurosci. 28, 1816-1823.

Vanhatalo, S., Palva, J. M., Holmes, M. D. Miller, J. W., Voipio, J., and Kaila, K. (2004). Infraslow oscillations modulate excitability and interictal epileptic activity in the human cortex during sleep. Proc. Natl. Acad. Sci. U.S.A. 101, 5053-5057.

Vanhaudenhuyse, A., Noirhomme, Q. Tshibanda, L. J. F., Bruno, M.-A. Boveroux, P., Schnakers, C., Soddu, A. Perlbarg, V., Ledoux, D., Brichant, J. F., Moonen, G., Maquet, P., Greicius, M. D., Laureys, S., and Boly, M (2010). Default network connectivity reflects the level of consciousness in non-communicative brain-damaged patients. Brain 133, 161-171.

Varoquaux, G., Sadaghiani, S., Pinel, P., Kleinschmidt, A., Poline, J. B., and
Thirion, B. (2010). A group model for stable multi-subject ICA on fMRI datasets. Neuroimage 51, 288-299.

Vincent, J. L., Kahn, I., Snyder, A. Z., Raichle, M. E., and Buckner, R. L. (2008). Evidence for a frontoparietal control system revealed by intrinsic functional connectivity. $J$ Neurophysiol. 100, 3328-3342.

Vincent, J. L., Patel, G. H., Fox, M. D., Snyder, A. Z., Baker, J. T., Van Essen, D. C., Zempel, J. M., Snyder, L. H., Corbetta, M., and Raichle, M. E. (2007). Intrinsic functional architecture in the anaesthetized monkey brain. Nature 447, 83-86.

Vyazovskiy, V. V., Cirelli, C., PfisterGenskow, M., Faraguna, U., and Tononi, G. (2008). Molecular and electrophysiological evidence for net synaptic potentiation in wake and depression in sleep. Nat. Neurosci. 11, 200-208.

Wackermann, J., Lehmann, D., Michel, C. M., and Strik, W. K. (1993). Adaptive segmentation of spontaneousEEG map series into spatially defined microstates. Int. J. Psychophysiol. 14, 269-283.

Waites, A. B., Stanislavsky, A., Abbott, D. F., and Jackson, G.D. (2005).Effect of prior cognitive state on resting state networks measured with functional connectivity. Hum. Brain Mapp. 24, 59-68.

Wyart, V., and Tallon-Baudry, C. (2009). How ongoing fluctuations in human visual cortex predict perceptual awareness: baseline shift versus decision bias. J. Neurosci. 29, 8715-8725.

Zarahn, E., Aguirre, G. K., and D'Esposito, M. (1997). Empirical analyses of BOLD fMRI statistics. I. Spatially unsmoothed data collected under nullhypothesis conditions. Neuroimage 5, 179-197.

Conflict of Interest Statement: The authors declare that the research was conducted in the absence of any commercial or financial relationships that could be construed as a potential conflict of interest.

Received: 05 February 2010; paper pending published: 07 March 2010; accepted: 12 May 2010; published online: 23 June 2010

Citation: Sadaghiani S, Hesselmann G, Friston KJ and Kleinschmidt A (2010) The relation of ongoing brain activity, evoked neural responses, and cognition. Front. Syst. Neurosci. 4:20. doi: 10.3389/ fnsys.2010.00020

Copyright $(2010$ Sadaghiani, Hesselmann, Friston and Kleinschmidt. This is an openaccess article subject to an exclusive license agreement between the authors and the Frontiers Research Foundation, which permits unrestricted use, distribution, and reproduction in any medium, provided the original authors and source are credited. 